\title{
On the Failure of Ellipticity for Compressible Isotropic Nonlinearly Elastic Materials
}

\author{
J. Merodio \\ Department of Continuum Mechanics and Structures, Escuela de Ingenieros de Caminos, Canales \\ y Puertos, Technical University of Madrid, 28040, Madrid, Spain
}

\begin{abstract}
The ordinary ellipticity status of some compressible isotropic nonlinearly elastic solids is examined under plane deformations.
\end{abstract}

Keywords: loss of ellipticity, differential equations, non-linear elasticity, isotropy

\section{INTRODUCTION}

In the past few years there has been a great deal of interest in determining the ordinary ellipticity status of the governing differential equations for either compressible or incompressible hyperelastic materials. In connection with asymptotic studies of crack problems, Knowles and Sternberg [1] found that the field equations of non-linear elastostatics might suffer a loss of ellipticity in the presence of severe local deformations. Conditions for ordinary ellipticity of the equilibrium equations are based on the nonsingularity of the acoustic tensor. Some of the consequences of the loss of ellipticity of the governing differential equations are well understood. It has been shown for a general compressible hyperelastic material in plane deformations that the loss of ellipticity is a necessary condition for the emergence of weak solution fields lacking the smoothness required by the differential equations. Among these weak solutions, the ones involving discontinuous deformation gradients, called equilibrium shocks, have received particular attention. The analysis of stress-induced phase transformations in continuummechanical modeling of solids also involves issues related to the ellipticity status of the deformations giving rise to the so called phase changes. There is also a notion of strong ellipticity of the equilibrium equations -based on the positive definitiveness of the acoustic tensor- but in this analysis the term ellipticity will refer to ordinary ellipticity. Here, the ellipticity status of some compressible isotropic nonlinearly elastic solids is examined under plane deformations. Constitutive equations that suffer a loss of ellipticity have been studied in a variety of contexts. In [2] the isotropic considered is a neo-Hookean material -incompressible-, while in [3], the isotropic used is the special Blatz-Ko material -compressible. The neo-Hookean model is known to keep elliptic at all deformations while the Blatz-Ko material is known to lose ellipticity at sufficiently large deformations both in tension and compression. The paper is organized as follows. In Section 2, a brief introduction on the parameters that characterize the material properties of the hyperelastic models under consideration and background information on the conditions for the governing differential equations to be elliptic are presented. In Sec- 
tion 3 , the procedure is shown examining the ellipticity status of a particular isotropic material are given. Section 4 is devoted to some conclusions.

\section{ISOTROPIC NONLINEARLY ELASTIC MATERIAL MODELS AND THE ELLIPTICITY OF THE EQUILIBRIUM EQUATIONS.}

Let $\mathbf{F}=\partial \mathbf{y} / \partial \mathbf{x}$ be the deformation gradient tensor, where $\mathbf{x}$ is the position vector of a material particle in the undeformed configuration and $\mathbf{y}$ is the corresponding position vector in the deformed configuration. The right Cauchy-Green deformation tensor, denoted $\mathbf{C}$, is given by $\mathbf{C}=\mathbf{F}^{T} \mathbf{F}$ and $I_{1}, I_{2}$ and $I_{3}$ are its principal invariants, which are given by

$$
\begin{gathered}
I_{1}=\operatorname{tr} \mathbf{C}, \\
I_{2}=\frac{1}{2}\left[(\operatorname{tr} \mathbf{C})^{2}-\operatorname{tr}\left(\mathbf{C}^{2}\right)\right], \\
I_{3}=\operatorname{det} \mathbf{C}=(\operatorname{det} \mathbf{F})^{2} .
\end{gathered}
$$

The most general strain energy density per unit undeformed volume for homogeneous isotropic nonlinear elastic solids is given by (See Ogden [4]) $W=W\left(I_{1}, I_{2}, I_{3}\right)$. Our concern in this paper is the ellipticity analysis in plane deformations of the materials introduced and that are modelled by that strain energy function. The compressible case will be considered. Without further detail let us introduce the equations of equilibrium and the notion of ellipticity of these equations. For planar deformations the displacement field $\mathbf{u}$ is of the form: $\mathbf{u}=\mathbf{y}-\mathbf{x}=u_{1}\left(x_{1}, x_{2}\right) \mathbf{i}_{1}+u_{2}\left(x_{1}, x_{2}\right) \mathbf{i}_{2}$ so that $F_{13}=F_{23}=F_{31}=$ $F_{32}=0$, and $F_{33}=1$. Therefore the components of $\mathbf{C}=\mathbf{F}^{T} \mathbf{F}$ obey

$$
C_{13}=C_{23}=0, C_{33}=1 \text {. }
$$

The governing partial differential equations of equilibrium for the displacement field $\mathrm{u}(\mathrm{x})$ in a homogenous compressible elastic material can be written as (See [1])

$$
\mathscr{C}_{\alpha \beta, \gamma \delta} u_{\alpha, \beta \delta}=0
$$

where

$$
\mathscr{C}_{\alpha \beta, \gamma \delta}=\frac{\partial^{2} W}{\partial F_{\gamma \delta} \partial F_{\alpha \beta}} .
$$

The quasi-linear system of differential equations (3) is elliptic at the solution $\mathbf{u}$ and at a point $\mathbf{x}$ if and only if $\operatorname{det}[\mathbf{Q}] \neq 0$ where

$$
Q_{\alpha \gamma}=\mathscr{C}_{\alpha \beta, \gamma \delta}(\mathbf{F}(\mathbf{x})) n_{\beta} n_{\delta}
$$

for every unit vector $\mathbf{n}=\left(n_{1}, n_{2}\right)$. $\mathbf{Q}$ is called the acoustic tensor and is symmetric. The ellipticity condition can be finally written as

$$
\operatorname{det}[\mathbf{Q}]=Q_{11} Q_{22}-Q_{12}^{2}=0 .
$$


The analysis of Eq.(8) for specific forms of the elastic potential $W$ gives the ellipticity status of that particular strain energy. Namely, a deformation gradient $\mathbf{F}$ satisfying Eq.(8), in case of compressible elastic models, for every unit vector $n$, is said to be an elliptic deformation for the elastic potential at hand. If all the deformations for a material are elliptic, then the material itself is referred as an elliptic one. On the other hand, if a deformation gradient $\mathbf{F}$, for any unit vector $\mathbf{n}$, does not satisfy Eq. (8) then the deformation is said to be non-elliptic for that material model. Furthermore, the unit vector $\mathbf{n}$ gives the normal vector to a direction, called weak elastostatic shock, across which, some of the differentiability requirements imposed in the derivation of the field Eqs.(5) are not satisfied by some or all the variables involved.

\section{THE CASE OF AN ISOTROPIC MATERIAL}

Let us analyze a simple case to show the procedure. Consider the isotropic compressible generalization of the neo-Hookean incompressible model

$$
W=\mu\left(I_{1}-3+E\left(I_{3}\right)\right) .
$$

The neo-Hookean material, given by the first term in (9), is known to remain elliptic at all deformations. The issue now is to establish some conditions on $E\left(I_{3}\right)$ to guarantee the ellipticity of the material model given by Eq.(9). Particular forms of $E\left(I_{3}\right)$ have been studied in [5] in the context of the generalized Blatz-Ko constitutive model. Furthermore, the ellipticity status of (9) follows easily from Lemma 5.2 of [6]. Nevertheless we will directly apply Eq.(6). First, we provide and prove the following proposition:

Proposition 1. If $\mathrm{M}$ and $\mathrm{N}$ are symmetric, and if $\operatorname{det} \mathrm{M} \geq 0$ with $M_{11} \geq 0$ and det $\mathrm{N}$ $\geq 0$ with $N_{11} \geq 0$, such that either $\operatorname{det} \mathrm{M} \neq 0$ or $\operatorname{det} \mathrm{N} \neq 0$, then $\operatorname{det}(\mathrm{M}+\mathrm{N})>0$. Proof:

$$
\operatorname{det}(M+N)>M_{11} N_{22}+M_{22} N_{11}-2 M_{12} N_{12} .
$$

It is sufficient to show that the right term in Eq.(10) is greater than zero. We will show it by contradiction. Assume that $M_{11} N_{22}+M_{22} N_{11}-2 M_{12} N_{12} \leq 0$, then $0 \leq M_{11} N_{22}+$ $M_{22} N_{11} \leq 2 M_{12} N_{12}$. Now, taking the square in both sides $\left(M_{11} N_{22}+M_{22} N_{11}\right)^{2} \leq$ $4 M_{12}^{2} N_{12}^{2}$. On the other hand, since det $\mathrm{M} \geq 0$ and det $\mathrm{N} \geq 0$, then $M_{11}^{2} N_{22}^{2}+M_{22}^{2} N_{11}^{2} \overline{+}$ $2 M_{12}^{2} N_{12}^{2} \leq\left(M_{11} N_{22}+M_{22} N_{11}\right)^{2} \leq 4 M_{12}^{2} N_{12}^{2}$. Whence, and by the same argument, $\left(M_{11} N_{22}-M_{22} N_{11}\right)^{2} \leq M_{11}^{2} N_{22}^{2}+M_{22}^{2} N_{11}^{2}-2 M_{12}^{2} N_{12}^{2} \leq 0$ which is only satisfied if and only if $\operatorname{det} \mathrm{M}=\operatorname{det} \mathrm{N}=0$. Therefore, $\operatorname{det}(\mathrm{M}+\mathrm{N})>0$. QED.

Based on the proposition, we restrict the ellipticity analysis of Eq.(9) to the ellipticity analysis of $E\left(I_{3}\right)$. By considering planar deformations and introducing Eq.(3) in $E\left(I_{3}\right)$, we find that the components of the acoustic tensor are

$$
\begin{gathered}
Q_{11}=E_{I_{3} I_{3}}\left(F_{11}^{-1} n_{1}+F_{11}^{-1} n_{2}\right)^{2} \\
Q_{22}=E_{I_{3} I_{3}}\left(F_{11}^{-1} n_{1}+F_{11}^{-1} n_{2}\right)^{2} \\
Q_{12}=E_{I_{3} I_{3}}\left(F_{11}^{-1} n_{1}+F_{11}^{-1} n_{2}\right)\left(F_{11}^{-1} n_{1}+F_{11}^{-1} n_{2}\right),
\end{gathered}
$$

where use of (1.20) of [7] has been made. Hence, det $Q_{E}=0$ and, by proposition 1, $E_{I_{3} I_{3}}(I 3) \geq 0$ is sufficient to guarantee the ellipticity of (9). By Lemma 5.2 of [6], the 
ellipticity analysis of (9) yields the necessary and sufficient condition $E_{I_{3} I_{3}}\left(I_{3}\right)>\frac{-2}{\beta^{2}}$, where $\beta>0$ depends on $\mathbf{F}$, for (9) to be elliptic. It is clear that the procedure followed here does not give that general condition on $E\left(I_{3}\right)$. Nevertheless, it will provide with a first approximation to the ellipticity analysis of (9) for particular forms of $E\left(I_{3}\right)$. For instance, consider the special Hadamard material

$$
W=\mu\left(I_{1}-3+\frac{1-2 v}{v} I_{3}^{\frac{v}{1-2 v}}+2-\frac{1}{v}\right) .
$$

By comparison with the material model given by Eq.(14) and since $E_{I_{3} I_{3}}\left(I_{3}\right)>0$ we can conclude that the constitutive model (14) remains globally elliptic under plane deformations.

The reason to consider positive definitive acoustic tensors is based on the concept of strong ellipticity. Although the ellipticity refers to the non-singularity of the acoustic tensor, the strong ellipticity refers to the positive definiteness of the acoustic tensor. A non-positive acoustic tensor in the undeformed configuration is not physically admissible.

\section{CONCLUSIONS}

Conditions for ordinary ellipticity of the equilibrium equations are based on the nonsingularity of the acoustic tensor. In the most general case, these conditions will be given as function of the derivatives of the strain energy function with respect to the invariants characterizing the material model. The breakdown of ellipticity gives both the deformation at which ellipticiy is lost and the surface of discontinuity characterized by the normal vector to the surface.

\section{REFERENCES}

1. J.K. Knowles and E. Sternberg, On the ellipticity of the equations of nonlinear elastostatics for a special material, J. Elasticity 5, 1975, 341-361.

2. L. Zee and E. Sternberg, Ordinary and strong ellipticity in the equilibrium theory of incompressible hyperelastic solids, Arch. Rat. Mech. Anal. 83, 1983, 50-90.

3. N. Triantafyllidis and R. Abeyaratne, Instability of a finitely deformed fiber-reinforced elastic material, J. Appl. Mech. 50 (1983) 149-156.

4. R.W. Ogden, Nonlinear Elastic deformations, Ellis Horwood Ltd (1984).

5. C.O. Horgan, Remarks on Ellipticity for the Generalized Blatz-Ko Constitutive Model for a Compressible Nonlinearly Elastic Solid, J. Elasticity 42, 1996, 165-176.

6. P. Rosakis, Ellipticity and deformations with discontinuous gradients in plane elastostatics of compressible solids, Arch. Rat. Mech. Anal. 109, 1990, 1-37.

7. J.K. Knowles and E. Sternberg, On the failure of ellipticity and the emergence of discontinuous deformation gradients in plane finite elastostatics, J. Elasticity 8, 1978, 329-379. 